\title{
El acontecer en el Aula Tecnológica y el aprendizaje de los alumnos*
}

Griselda Constantino**

\begin{abstract}
The Technical and Professional Education Council of Uruguay (also known as Work University) offers the graduates from all the primary schools of the country the possibility of attending the "Ciclo Básico Tecnológico" (Technological Basic Cycle). Besides the traditional subjects this cycle includes the technological learning through a subject called "Área Tecnológica" (Technological Area), with the elaboration of projects that are developed throughout the year.

This research aims at achieving a throughout understanding of the Aula Tecnológica as well as its possible influences on good learning-strategies. From a qualitative-interpretative approach, it enquires into the relationship between teacher and student, the syllabus proposal, the learning methods, the didactic resources, and the physical space where everything takes place. In this case in particular, at the Escuela Técnica de Colonia del Sacramento (Technical School at Colonia del Sacramento).
\end{abstract}

As a conclusion, the technological area is seen as a bridge between theory and practice, where 'learning-by-doing' is what leads the work.

\section{RESUMEN.}

El Consejo de Educación Técnico Profesional ofrece a los alumnos egresados de las escuelas de nuestro país, la posibilidad de ingresar a una propuesta de estudio en el Ciclo Básico Tecnológico, que incluye además de las asignaturas tradicionales el componente tecnológico, a través de un espacio denominado Aula Tecnológica con la elaboración de proyectos que se llevan a cabo durante el año lectivo.

En este trabajo de investigación se intenta entender el Aula Tecnológica en todo su acontecer, y sus posibles influencias en buenas estrategias de aprendizaje. Se indaga desde un enfoque cualitativo-interpretativo especialmente la relación docente-alumno, la propuesta programática ofrecida, sus métodos de enseñanza, los dispositivos didácticos y el espacio físico en el que todo ello transcurre, inmerso en este caso particular en la Escuela Técnica de Colonia del Sacramento.

Se concluye en que el aula tecnológica se reconoce como un lugar de puente entre la teoría y la práctica en la cual es el aprender haciendo el que guía el trabajo.

\section{El aula tecnológica: su forma de trabajo y su vinculación con el aprendizaje de los alumnos}

La presente investigación ha tenido como objeto las estrategias de enseñanza que introducen los docentes en el aula tecnológica de la Escuela Técnica y su vinculación con el aprendizaje de los alumnos.

Nos ha interesado conocer de qué forma las estrategias de enseñanza de los docentes del aula tecnológica potencian la relación docente-alumno y cómo intervienen para lograr un buen aprendizaje en los alumnos. En este sentido algunas de las preguntas que han orientado el 
proceso de investigación son: ¿qué métodos utilizados favorecen el buen aprendizaje en la educación tecnológica?, ¿qué concepciones de tecnología tienen los docentes del área tecnológica de la Escuela Técnica de Colonia?, ¿qué acciones didácticas o pedagógicas se llevan adelante en el aula tecnológica para que los alumnos logren un buen aprendizaje?

El objetivo que ha guiado la investigación ha sido el de reconocer las estrategias de enseñanza de los docentes del aula tecnológica e indagar si potencian los aprendizajes de los alumnos.

Nos hemos propuesto además:

- Reconocer las estrategias de trabajo utilizadas en el aula.

- Identificar las formas de intervención docente y su relación con los aprendizajes.

- Describir la organización y utilización del espacio físico del aula tecnológica.

- Analizar las metodologías y dispositivos y construir categorías que permitan entrecruzar la enseñanza de la tecnología y el aprendizaje en el contexto del aula tecnológica.

El trabajo de investigación ha apuntado, por tanto, a comprender las relaciones entre las estrategias de enseñanza y los aprendizajes de los estudiantes en el aula tecnológica.

Siendo el aula tecnológica el elemento que distingue la propuesta educativa de las Escuelas Técnicas en el ciclo básico es que nos ha interesado conocerla en relación con los aprendizajes logrados por los alumnos.

Desde que egresé del instituto de formación he tenido la oportunidad de trabajar como docente en diferentes Escuelas Técnicas del departamento, y así he podido acercarme a la propuesta que plantea el aula tecnológica, y me ha parecido pertinente y relevante profundizar en las estrategias y el tipo de conocimiento que se genera desde el aula tecnológica, motivo por el cual me he embarcado en esta investigación.

Ha sido mi interés indagar sobre la forma en que es concebida la tecnología desde este espacio educativo, para analizar a la luz del marco referencial que le da origen a la propuesta programática, el planteo educativo que allí se realiza. Como es de esperar hay algunos supuestos que han sido parte de la investigación. Entre ellos el supuesto de que el desarrollo de un país no está condicionado por sus recursos naturales, sino por la capacidad de transformarlos en bienes de alto valor agregado en la cadena productiva, y es justamente el aporte tecnológico quien lo hace posible.

La ciencia y la tecnología se han incorporado a todos los aspectos de la vida del hombre, y por eso la necesidad de brindar educación tecnológica acorde a los tiempos que corren, desde un enfoque transversal, porque los conocimientos tecnológicos están condicionados histórica y socialmente y también ellos condicionan la vida del hombre.

Se pretende enfocar la enseñanza de la tecnología como un saber práctico en relación con los conocimientos teóricos, y que además puedan aplicarse en la producción de bienes y servicios para solucionar problemas o responder a necesidades humanas.

El aula tecnológica se concibe como un salón-taller, que tiene las dimensiones, características, comodidades y herramientas propias de un taller, pero que también cuenta con un pizarrón y un escritorio para los docentes.

Los alumnos trabajan en mesas con los compañeros de su equipo de pares cuya integración se organiza al comienzo del año lectivo y disponen de taburetes para sentarse. Son los propios alumnos además los encargados de preocuparse por el orden y la prolijidad del salón-taller. 
La metodología que se emplea es la resolución de problemas a través de la realización de un proyecto tecnológico, definido en la fundamentación del plan vigente y cuya última modificación corresponde al año 2007, con las ideas que se presentan a continuación aunque no en forma textual, pero que hacen referencia a los proyectos tecnológicos como el proceso y el producto resultante que tienen como objetivo la creación, modificación y/o concreción de un producto, o la organizacióny/o plantificación de un proceso.

En las diferentes fases del proyecto tecnológico, hay que tener en cuenta todos los factores que pueden condicionar o incidir en el producto, los cuales pueden ser técnicos,tecnológicos, socioculturales o económicos.

En este sentido el trabajo de tesis me ha permitido, de alguna manera, resignificar mi propia mirada sobre las formas del enseñar y del aprender en el contexto del aula tecnológica y revisar mis propios supuestos desde una mirada analítica y teórica.

\section{UNA APROXI MACI ÓN CUALITATIVA: EL CLI MA DE TRABAJO EN EL AULA TECNOLÓGI CA}

Las encuestas han dado cuenta de diferentes modos en que los estudiantes encuentran placentero el trabajo en el aula tecnológica. Por un lado, por la modalidad de taller y de producción; por una mirada docente atenta que otorga valor al trabajo del alumno y fortalece su sentido de agencia (Bruner, 1997).

Desde una interpretación cualitativa y segun la mirada analítica sobre los datos, hemos reconocido que los alumnos manifiestan su agrado por trabajar en el aula tecnológica, prefiriéndolo como espacio físico para realizar sus tareas más que el salón que sigue respondiendo al modelo vareliano, organizado con bancos uniformemente dispuestos, por los cuales el alumno tiene como línea de horizonte la nuca de su compañero y le dificulta la movilidad, el trabajo en equipo y el intercambio, tan oportunos para favorecer los procesos de aprendizaje.

En la modalidad de trabajo del aula tecnológica uno de los aspectos bien interesantes a señalar es que los docentes trabajan en equipo, constituyen una pareja pedagógica ya que dos docentes son los encargados del grupo, trabajan en simultáneo, e incluso según pudimos saber por el relato realizado por el coordinador cuando el plan se creó en el año 1997, estaba previsto que los equipos de trabajo fueran de tres docentes que pertenecieran a diferentes áreas, para de esta manera complementarse en el trabajo, para que existiera más amplitud y oportunidades diversas para los alumnos en el momento de elegir su proyecto o señalar sus centros de interés, y que pudieran contar con el asesoramiento y la guía necesaria para llevarlos adelante de la mejor forma posible. Si bien hoy esto no es así, se ha desvirtuado el espíritu que el plan tenía en este aspecto para responder a necesidades laborales, dejando que los docentes opten por la escuela o los grupos en los que les queda mejor trabajar sin que se constituya en impedimento el que dos docentes pertenezcan a la misma área.

De todas maneras, en el caso de la escuela en que se realiza la investigación, la pareja pedagógica cumple con esta característica de ser un docente egresado de la escuela de mecánica y el otro de la de carpintería. Al respecto éste último nos decía; "trabajamos en simultáneo los dos docentes sí, porque como tenemos distintos perfiles -el perfil mío es de base de carpintería y el otro compañero que trabaja conmigo es mecánico-, se combinan y vamos manejando los temas según nuestra especialidad". (1)

Resulta significativo que en el aula tecnológica trabajen dos docentes juntos a cargo del mismo grupo conformando una pareja pedagógica que comparte objetivos, planificaciones, modalidades 
de trabajo, acuerdos áulicos, pero este aspecto sólo es mencionado en contadas ocasiones por los alumnos.

Una primera interpretación podría dar cuenta de que el trabajo en pareja pedagógica muestra mayor atención, mayor contención. No obstante, no es apreciado así por los alumnos, o quizás el hecho se ha naturalizado e incorporado al ritual áulico del taller. En este sentido, disiente la mirada desde los docentes con las apreciaciones de los estudiantes.

El hecho anteriormente mencionado se corrobora con la observación de clase ya que uno de los docentes permanece sentado en su escritorio la mayor parte del tiempo, por lo que tal vez los alumnos no aprecien sus aportes académicos o sus intervenciones en la construcción del conocimiento.

El docente que ocupa el cargo de coordinador del aula tecnológica desde el año 1997 al momento de realizarse la investigación cuenta con una experiencia de 11 años y además, 24 años de experiencia como maestro técnico, efectivo, grado 7, factores que nos indican muy importantes insumos vivenciales. También recibió formación específica para trabajar según la modalidad que plantea el aula tecnológica durante 6 años en la capital del país, favoreciendo así la diversidad de miradas y enfoques, entre los docentes del aula tecnológica.

Es interesante señalar que muchas veces sucede que el espíritu, la intención que fue puesta en la elaboración de los programas, básicamente en su fundamentación, no se materializa en el acto educativo una vez que la propuesta es llevada adelante por el docente.

La modalidad de trabajo en pareja pedagógica en un aula con las características que hemos señalado constituyen el aula tecnológica, tiene las siguientes virtudes: atención más personalizada, protección frente a diversos peligros propios del uso de herramientas que se utilizan; atención inmediata a los emergentes que puedan llevar a perder el trabajo de todo el equipo por una inexactitud de centímetros, especificidad en la orientación del proyecto teniendo en cuenta que ambos docentes pertenecen a diversas áreas, amplitud en el planteo teórico y práctico del problema, considerando esta diferente especificidad que aporta al enriquecimiento del proyecto en su conjunto, respuesta más rápida y eficaz del docente a cada equipo.

Los alumnos trabajan en grupos sentados alrededor de diferentes mesas distribuidas en el taller, debiendo el docente sólo en casos excepcionales cambiar los integrantes, después de haber sido invitados a pensar que "cuando el río esta picado, no hay que abandonar el bote sino remar juntos para llegar a la orilla" (metáfora utilizada por uno de los docentes observados y entrevistados).

Se desprende de las encuestas e incluso de las entrevistas realizadas a los alumnos, el gusto por el trabajo en modalidad de proyecto, al punto tal que utilizan la palabra proyecto para referirse no sólo a la forma de trabajo empleada en el aula tecnológica sino también, la que constituye la actividad que más les gusta realizar, sin precisar un momento específico del trayecto que involucra trabajar con dicha modalidad, sino como si en sí mismo el proyecto fuese una actividad de aula.

Esta idea se refuerza con lo expresado por los alumnos al decir no recordar o no ser conscientes de haber trabajado antes, desde la escuela, con el método de proyectos que, sabido es, quizás no con la formalidad que se usa en el aula tecnológica, pero si es usado fundamentalmente en ciencias en la escuela primaria.

En síntesis, reconocemos que se han naturalizado ciertas prácticas y modos de trabajo. Esta naturalización, no obstante, no obstruye la manifestación del placer por estos modos de abordaje en el aula. 


\section{METODOLOGÍ A}

La investigación se enmarca en la lógica cualitativa en tanto el objeto consiste en reconocer determinadas prácticas en contexto e interpretarlas a la luz de desarrollos teóricos derivados de la didáctica, el campo de la tecnología educativa y la psicología.

La investigación cualitativa tal como lo expresa el autor Eisner (1998) influye en los hechos cotidianos de la vida diaria. El pensamiento cualitativo...."se extiende por nuestros juicios diarios y proporciona las bases para nuestras investigaciones más importantes"...(2) porque desde el nacimiento y a lo largo de nuestra vida le asignamos cualidades y aun antes las experimentamos a través de los sentidos, para luego establecer categorías.

En este caso se conocerán los dispositivos didácticos, las intervenciones docentes en las situaciones de enseñanza y aprendizaje en el aula tecnológica, para desde el reconocimiento de su metodología y estrategias comprender el aprendizaje que los alumnos logran, y su aplicación a situaciones nuevas e incluso en otras áreas o ámbitos de trabajo.

Se trata de ahondar en el uso del espacio educativo que denominamos aula tecnológica, en vista a comprender las prácticas educativas y a poder pensar en mejores aprendizajes potenciados por los docentes del aula tecnológica.

Según lo planteado por Salkind en "Métodos de Investigación", es de tipo no experimental, descriptiva, porque reseña características de un fenómeno existente, no de un único caso sino del alumnado de un grupo perteneciente al Ciclo Básico Tecnológico de la Escuela Técnica de Colonia, considerado en el aula tecnológica.

El docente en ese "rol extendido" que ha asumido en las últimas décadas, además de contener, formar, facilitar, también constantemente es un explorador en búsqueda de los aspectos que a simple vista se presentan solapados, escurridizos, escondidos, y que pudieran informarnos sobre lo que a nuestro alumno le ocurre, sobre lo que necesita, sobre su "situación real".

No debemos olvidar que los significados se construyen; que tienen que ver con los métodos que usemos para llegar a ese caudal informativo, y que son miradas de un hecho o asunto pero que también hay otras igualmente válidas.

Para intervenir en las situaciones de aprendizaje que se producen en un espacio áulico, necesitamos saber sobre su contexto, su historia, sus cualidades, características del todo (el espacio) y de cada uno de los participantes, porque este conocimiento nos ayudará a interpretar los datos. Sin duda algo de esto intentamos los docentes cada año al comenzar el año lectivo elaborando fichas personales, participando de la reunión de antecedentes, acercándonos al equipo multidisciplinario en el caso de que nuestra institución educativa lo tenga, y es en este sentido, que en esta investigación se conocerá el acontecer en el aula tecnológica en relación con las propuestas programáticas, los métodos utilizados, los dispositivos didácticos, la relación docentealumno, las características áulicas, los instrumentos de trabajo, el aprendizaje de los alumnos, para entender su múltiple influencia.

Algunas otras características de la investigación que se realiza son:

- Seccional en relación con el alcance temporal, o sea un estudio transversal porque se observa y analiza un proceso en un momento determinado (año lectivo 2008).

- De profundidad descriptiva porque da cuenta de las características de los fenómenos, del acontecer, referido a personas en un lugar y un tiempo.

- De utilización de fuentes mixtas primarias y secundarias.

\section{TÉCNI CAS UTI LI ZADAS}

Para esa investigación se realizaron entrevistas semiestructuradas a los docentes del aula tecnológica, teniendo en cuenta primero el propósito de la entrevista según las metas del proyecto.

Se elaboraron algunas preguntas estructuradas de antemano y otras surgieron en la propia entrevista, pero teniendo en cuenta que debían ser formuladas desde los aspectos más sencillos 
a los más complejos y de forma clara y directa para evitar confusiones. Las entrevistas fueron realizadas en la propia aula tecnológica, ambiente por naturaleza muy cómodo para los docentes y para el Coordinador del Área Tecnológica en horario extraescolar.

También se realizó una encuesta a alumnos de 1er año del Ciclo Básico Tecnológico para conocer la concepción, la idea, la representación del aula tecnológica y su utilidad para ellos mismos y para su aprendizaje, además de las motivaciones que pueda presentar esa modalidad de trabajo. El universo de encuestados comprendió a todos los alumnos de 1er año o sea un total de 120 estudiantes a quienes se les consultó con preguntas o afirmaciones para que seleccionaron la opción que preferían en forma clara y concisa, comenzando con las más generales y luego las más específicas.

Otra de las técnicas utilizadas y de vital importancia, fue la observación de clase no participativa, de la que se hizo un registro escrito pormenorizado y se grabó el audio, para poder describir y comprender al grupo protagonizando sus vivencias en el aula tecnológica.

Eisner (1998) expresa: “La observación científica consiste en un proceso sistemático, deliberado y selectivo por el cual un investigador obtiene información relativa a una situación o problema; es fundamental en la investigación cualitativa y proporciona una representación de la realidad en la que intervienen la percepción y la interpretación del observador". (3)

La metodología implementada en esta investigación incluye la observación de clases en el aula tecnológica, en el taller como lugar de construcción del conocimiento, desde la cooperación, por su formato, por sus características espaciales, por la manera en que está concebido en cada institución educativa.

De la primera observación realizada a una clase del grupo $1^{\circ} \mathrm{C}$ en el aula tecnológica, se pudieron inferir algunas consideraciones que permitieron clasificar los sucesos que son parte del acontecer de la clase, lo vivido por los diferentes actores del acto educativo, y otros que corresponden a las impresiones y sensaciones percibidas por el observador en una estrategia de observación no participativa.

Una vez realizada la encuesta a la totalidad de los alumnos de 1er y 2do año del ciclo básico tecnológico y comenzando con la tarea de leer las mismas para sistematizar los resultados, se hace evidente la necesidad de repreguntar, de profundizar en algunos aspectos que de acuerdo a las características de la técnica usada (encuesta) resultaba escasa la información y seguramente muy pertinente insistir en aspectos claves para la investigación.

Por tal motivo se resuelve agregar entrevistas a 9 alumnos del grupo $1^{\circ} \mathrm{C}$ al cual acompaño los viernes en sus clases cuando realizo la observación no participativa, entendiendo que el hecho de que los alumnos me vean habitualmente en su salón de clase, que conocen que estoy realizando una investigación y no calificando sus actuaciones, favorecerá para que sus respuestas sean sinceras y claras.

Si bien en lista figuran 24 alumnos, el día que se realiza la entrevista asisten a clase 18 alumnos, por lo cual se opta por entrevistar a 9 de ellos tanto varones como chicas para que sea representado el $50 \%$ del grupo asistente a clase. Las entrevistas se realizan en un salón de clase del centro educativo en horario escolar, ingresando los alumnos en tríos para favorecer su desenvolvimiento, para que no se sientan cohibidos sino que cómodamente puedan responder a las preguntas formuladas sobre su trabajo en el aula tecnológica.

La entrevista fue diseñada al estilo semiestructurada, con breves preguntas para no cansar a los alumnos y lograr la mejor actitud y clima de trabajo, lo que se evidenció en su interés en ser entrevistados, incluso algunos de ellos manifestaban lo relevante que era para ellos poder formar parte del trabajo de investigación que se estaba realizando. 
Cabe señalar que al momento de realizar las entrevistas a los docentes, también se buscó crear el ambiente más cómodo y oportuno, por lo cual uno de los docentes y el coordinador fueron entrevistados en el taller, en la propia aula tecnológica, en su lugar físico de trabajo, y el otro docente en el salón de clase que usaban para las clases teóricas porque los talleres estaban ocupados con clase el día y a la hora previstos para la entrevista.

\section{CONCLUSI ONES DE LA I NVESTI GACIÓN}

En la presente investigación hemos indagado acerca de cómo se aprende en el aula tecnológica. Como toda investigación reconocemos que no hemos acabado las preguntas ni encontrado todas las respuestas, sobre el aprendizaje o acerca de qué estrategias didácticas introducen los docentes para trabajar en dicha aula, pero hemos descrito un escenario en donde se produce el acto educativo con sus singularidades, en un contexto determinado, y podemos hacer algunas precisiones al respecto, como también señalar otros caminos a recorrer, siendo llevados por el deseo de conocer cómo se aprende y cómo se enseña en el aula tecnológica, para lograr una buena enseñanza.

En esta investigación hemos pretendido describir e interpretar los fenómenos educativos con un enfoque holístico y contextualizado, tratando de entender el trabajo del aula tecnológica y su alcance en los procesos constructivos del alumno.

Jackson (2002:34) sostiene que hay muchas maneras de actuar para lograr una buena enseñanza y Litwin (2008:27) señala que los mecanismos por los cuales elegimos una estrategia y descartamos otras para un tema y en un momento determinado, "siguen siendo una interrogante potente" (4), porque de esta decisión depende aquello de una buena enseñanza, máxime si tenemos en cuenta que los grupos son heterogéneos y las diferentes situaciones harán que surjan emergentes diversos, no pudiendo sostener una clase en forma idéntica a otra, aunque se utilice la misma planificación de base, aunque se intente aplicar la misma estrategia.

El aula tecnológica se configura como un espacio de aprendizaje que tiene su identidad propia, y dicha identidad se construye en base a ciertas pautas de funcionamiento que le son propias, y que en ciertos casos no condicen con las de la institución educativa que las contiene y de la cual forman parte.

Esta aparente arbitrariedad de actuación en el proceder puede derivar de dos fuertes improntas. Una está relacionada con el hecho de que los docentes y el coordinador son expertos, con varias décadas de desempeño docente y con el absoluto convencimiento de que la forma de trabajo y las estrategias utilizadas son las más convenientes, no sólo para el aula tecnológica, sino incluso debieran ser adoptadas y utilizadas por todos los docentes. La otra, con la fuerza soslayada que le dio origen como escuela para recuperar jóvenes de hábitos inadecuados para la vida social, y reeducarlos en un oficio que los insertara en forma "útil" en esa sociedad a la que pertenecían y que poseía un fuerte espíritu disciplinador.

La estrecha relación, entre la aplicación del método científico para solucionar problemas planteados a los alumnos, contextualización al medio, y fundamentalmente el hacer como camino posible para llegar al aprendizaje significativo, es lo proclamado sin cansancio por los docentes que tienen en alta estima todas las estrategias utilizadas desde el aula tecnológica y desvalorizan el trabajo teórico, lo producido por la mente, por la inmediata asociación a actividades aburridas, monótonas. Parecería que se olvida el placer de la lectura, de la búsqueda y la posibilidad de construir desde lo cognitivo, desde lo teórico, se olvidan las múltiples posibilidades de construir y hacer que no pasan necesariamente por lo manual, que no implican ruido y trabajo en taller, sino que se pueden lograr desde el pensamiento, desde la metacognición. 
Hay en el discurso de los docentes del aula tecnológica una fuerte intención de preparar operarios para el mundo del trabajo, aquello de insistir permanentemente en el uso correcto de las herramientas de trabajo, en el orden que implica usarlas y devolverlas al tablero para que todo quede como al comienzo de la clase, la higiene del taller, el colocar los taburetes sobre los mesas al retirarse los alumnos del taller, aunque al ingresar el siguiente grupo, lo primero que harán será volver a bajarlos de las mesas para poder trabajar, pero en esta ceremonia, están fuertemente implícitas las ideas de orden e higiene que se requieren en una fábrica. Se termina el día de aprendizaje cual día de trabajo, y es necesario en un cierre de actividad que no queden puertas abiertas para el otro equipo, cabos sin atar, todo en perfecto orden, y para ello, se destinan diez minutos de clase previo al final para las mencionadas tareas.

Desde las entrevistas se puede apreciar cómo los docentes sienten y piensan que preparan para la vida, lo que le va a ser útil al muchacho cuando egrese, pero cargando a esa palabra "útil" de una conceptualización unida específicamente al mundo del trabajo. Tengamos en cuenta que los docentes referidos han iniciado su labor docente cuando el diseño educativo de la institución tenía como finalidad preparar operarios para un país que intentaba industrializarse para responder a las demandas del mercado mundial (primera mitad del siglo XX), Universidad del Trabajo del Uruguay como reza su nombre.

Hoy, que las Escuelas Técnicas del país han diversificado sus propuestas y contienen también la posibilidad de que el alumno egrese de ciclo básico y continúe sus estudios en bachillerato, y luego acceda a la universidad para hacer una carrera de corte academicista, no debemos olvidar que continúa estando presente el componente técnico, ya que se denominan bachilleratos tecnológicos los ofrecidos por las Escuelas Técnicas y se pretende en cada una de sus orientaciones que el alumno básicamente en 3ero, a la vez de realizar sus estudios, tenga la oportunidad de hacer pasantías en empresas privadas o entes públicos como primera experiencia laboral.

En posteriores trabajos de investigación sería interesante, dándole más tiempo a esta experiencia, indagar sobre las repercusiones que esta estrategia ha tenido en los ámbitos en los cuales se produjo y en el proceso de aprendizaje de los alumnos involucrados. Podríamos suponer que aquello del contrato fundacional que da origen a las instituciones hace nuevas apariciones en cada innovación programática que se produce, no pudiéndonos desprender del matiz que nos dio origen, o no queriendo perder ese matiz porque se considera lo útil y necesario para la formación de nuestros jóvenes, para el país que queremos. Si bien la educación no es la única responsable en la formación del destino de cada país, tiene un aporte fundamental, en tanto y en cuanto, quienes tomarán las decisiones políticas en el futuro, tomando esta palabra en su más amplio sentido, serán algunos de los jóvenes que hoy están siendo formados en algunas de las aulas tecnológicas bajo las premisas educativas que se han considerado en la presente investigación.

Quizá la reflexión que podemos realizar a esta manera de considerar el aprendizaje y la enseñanza, es que existen múltiples maneras de conocer el mundo; el saber se hace y se descubre, las representaciones de lo que nos rodea se construyen y no hay un único camino.

La importancia de este tipo de enseñanza técnica tiene que ver con que no todos seguimos carreras universitarias, pero sí, todos en algún momento de nuestras vidas tendremos que trabajar, ingresar al mercado laboral, sin duda en algún momento de nuestras vidas nos enfrentaremos a un manual de algún artefacto que nos requiera decodificar lenguaje técnico.

En síntesis hemos encontrado que:

- El aula tecnológica se reconoce como un lugar de puente entre la teoría y la práctica en la cual es el aprender haciendo el que guía el trabajo;

- La estrategia de trabajo en modalidad de proyectos acerca al alumno con su docente, 
potenciado por la carga horaria de cinco horas semanales destinadas para el trabajo en el aula tecnológica;

- El hecho de trabajar en pareja pedagógica facilita mayores y mejores tiempos para el aprendizaje, en tanto la atención a cada equipo de trabajo puede ser más detenida, pausada;

- Los docentes entienden que la tecnología permite la resolución de problemáticas de la vida cotidiana que se materializan en la concreción de un trabajo, artefacto, y esta idea optimista, de confianza en la tecnología como facilitadora de la vida del hombre, es trasmitida constantemente al alumno en forma explícita o implícita;

- El trabajo en equipo, integrado además de forma heterogénea, estimula al alumno a negociar desavenencias y a resolver conflictos de manera pacífica, lo cual es necesario en todos los órdenes de la vida;

- El estilo de trabajo en el aula tecnológica, así como su entorno físico y su mobiliario, favorecen la potenciación de vínculos cooperativos de trabajo entre pares y con el docente que media dichos encuentros;

- Los alumnos encuentran el trabajo en el aula como placentero porque se logra un clima de trabajo muy distendido y porque el centro del curriculum en torno al cual se desarrollan todas las actividades áulicas hace referencia a un problema real, contextualizado, que les plantea el desafío de crear, hacer, para encontrarle una solución factible;

- Los docentes están convencidos de que el método y estrategia de trabajo por ellos utilizados son laos únicos válidos y debieran ser utilizados por todos los docentes sin excepción;

- El hacer, como único camino posible para llegar al aprendizaje significativo, es la concepción que se desprende de lo expresado por los docentes;

- Hay en el discurso de los docentes del aula tecnológica, una fuerte intención de preparar operarios para el mundo del trabajo;

- En las entrevistas se puede apreciar cómo los docentes sienten y piensan que desde su lugar preparan para la vida, aportan mucho más que los conocimientos de una asignatura, facilitan las competencias y habilidades necesarias para la vida misma.

\section{CITAS BIBLI OGRÁFICAS}

${ }^{1}$ Entrevista al profesor del aula tecnológica, profesor Washington Alvarez

${ }^{2}$ EISNER, E. 1998. El ojo ilustrado. Indagación cualitativa y mejora de la práctica educativa. Barcelona: Paidós.

${ }^{3}$ EISNER, E. 1998. El ojo ilustrado. Indagación cualitativa y mejora de la práctica educativa. Barcelona: Paidós.

${ }^{4}$ LITWIN, Edith. 2008. El oficio de enseñar. Condiciones y contextos. Buenos Aires: Paidós.

\section{BI BLI OGRAFÍ A}

BRUNER, J. 1997. La Educación: Puerta de la Cultura. Madrid: Visor.

BRUNER, J. 1998. Desarrollo cognitivo y educación. Madrid: Morata.

BUCKINGHAM, D. 2008. Más allá de la tecnología. Aprendizaje infantil en la era de la cultura digital. Buenos Aires: Manantial.

CARRETERO, M. et al. 1998. Procesos de enseñanza y aprendizaje. Madrid: Aique.

CARBONELL, J. 2001. La aventura de innovar. El cambio en la escuela. Madrid: Morata.

C.E.T.P. 2007. Educación y Arte Pedro Figari Montevideo: Escuela de Artes Gráficas.

CORNU, L. 1999. La confianza en las relaciones pedagógicas. Buenos Aires: Novedades Educativas. 
DABAS, E. 1998. Los contextos del aprendizaje. Situaciones socio-psico-pedagógicas. Buenos Aires: Nueva Visión.

DAVINI, M. C.1996. La formación docente en cuestión: política y pedagogía. Buenos Aires: Paidós.

DE LA TORRE, S. y BARRIOS, O. (coord.). 2000. Estrategias didácticas innovadoras. Recursos para la formación y el cambio. Barcelona: Octaedro.

EISNER, E. 1998. El ojo ilustrado. Indagación cualitativa y mejora de la práctica educativa. Barcelona: Paidós.

EISNER, E. 1998. Cognición y currículum. Una visión nueva. Buenos Aires: Amorrortu.

ELLIOT, J. 1993. El Cambio Educativo desde la Investigación-Acción. Madrid: Morata.

FERNANDEZ, L. 1994. Instituciones educativas. Dinámicas institucionales en situaciones críticas. Buenos Aires: Paidós.

FERRÉS, J. 2000. Educar en una cultura del espectáculo Barcelona: Paidós.

HANSEN, D. 2001. Llamados a Enseñar. Madrid: Idea Books.

ICART, M. T. 2006. Elaboración y presentación de un proyecto de investigación y una tesina. Barcelona: Edición Universitat de Barcelona.

JACKSON, P. 1999. Enseñanzas implícitas. Buenos Aires: Amorrortu.

KACHINOVSKY, C. 1988. Observaciones sobre la Inteligencia. Su exploración y revisión critica. Montevideo: Roca Viva.

KINCHELOE, J. 2001. Hacia una revisión crítica del pensamiento docente. Barcelona: Octaedro.

KUHN, TH. 1971. La Estructura de las Revoluciones Científicas. México: FCE.

LISTON, D. 1997. Formación del profesorado y condiciones sociales de la escolarización. 2da. ed. Buenos Aires: Morata.

LITWIN, E. (comp) 2005. Tecnologías educativas en tiempos de Internet. Buenos Aires: Amorrortu.

LITWIN, E. 2008. El oficio de enseñar. Condiciones y contextos. Buenos Aires: Paidós.

MARTI, E. 2005. Desarrollo, cultura y educación. Buenos Aires: Amorrortu.

MARTINEZ MONTERO, A. et al. 1967. Historia de la Universidad del Trabajo del Uruguay. Montevideo: Escuela de Artes Gráficas.

MEIRIEU P. 2001. La opción de educar. Ética y pedagogía. España: Octaedro.

MEIRIEU P. 1992. Aprender, Sí pero ¿cómo? Barcelona: Octaedro.

MORIN, E. 2001. Los Siete Saberes Necesarios para la Educación del Futuro. Buenos Aires: Nueva Visión.

PARDINAS, Fe. 1996. Metodología y Técnicas de Investigación en Ciencias Sociales. 36 Ed. Montevideo: Siglo XXI.

PERKINS, D. 1995. La escuela inteligente. Del adiestramiento de la memoria a la educación de la mente. Barcelona: Gedisa.

POZO MUNICIO, J.1996. Aprendices y Maestros. La Nueva Cultura del Aprendizaje. Madrid: Alianza. 
POZO, J. 2001. Humanamente. El mundo, la conciencia y la carne. Madrid: Morata.

PRIETO, D. 2005. La comunicación en la educación. Buenos Aires: Stella.

RESNICK, L. 1996. Curriculum y cognición. Buenos Aires: Aique.

REYES ABADIE, W. et al. 2000. Crónica General del Uruguay. Montevideo: Banda Oriental. SALKIND, N. 1999. Métodos de Investigación. 3era ed. México: Pearson Educación.

SCHVARSTEIN,FALTA INICIAL 1991. Psicología social de las organizaciones. Nuevos aportes. Buenos Aires: Paidós.

SIERRA BRAVO, R. 1988.Técnicas de Investigación Social. Teoría y Ejercicio. Madrid: Paraninfo.

SIERRA BRAVO, R. 1984. Ciencias Sociales. Epistemología, Lógica y Metodología. Teoría y Ejercicios. Madrid: Paraninfo.

STAKE, R .1998. Investigación con Estudios de Casos. Madrid: Morata.

TORP L. et al. 1998. El aprendizaje basado en problemas. Desde el jardín de infantes hasta el final de la escuela secundaria. Buenos Aires: Amorrortu.

VALLES, M. 1997. Técnicas Cualitativas de Investigación Social: reflexiones metodológicas y práctica profesional. Madrid: Síntesis.

WAINERMAN, C. et al. comp. 1997. La Trastienda de la Investigación. Buenos Aires: Lumière.

* El artículo se enmarca en la tesis de maestría de la autora, dirigida por la Dra. Carina Lion. Año 2009.

** Master en Educación, Universidad ORT Uruguay. Diploma en Educación, Universidad ORT Uruguay. Profesora de Educación Media -Especialidad Historia, Instituto Cerp del Suroeste. Docente en Consejo de Educación Técnico Profesional y Consejo de Educación Secundaria. 